\title{
Development of System for Visualizing of Phenomenon up to Ultimate State of Existing Structures with Image Information Technology
}

\author{
Masayoshi WADA \\ Engineer \\ Japan Information \\ Processing Service Co.,Ltd. \\ Osaka, Japan
}

Masayoshi Wada, born 1976, graduated from Nagasaki Univ. 1999, and got his Master of Engineering from Nagasaki Univ. 2001.

\section{Takashi YAMAGUCHI Assistant Professor Osaka City University Osaka, Japan}

Takashi Yamaguchi, born 1968, graduated from Kyoto Univ. 1991, and got his Doctor of Engineering from Kyoto Univ. 1996.

\author{
Masanori KITAMURA \\ Graduate Student \\ Osaka City University \\ Osaka, Japan
}

Masanori Kitamura, born 1977, graduated from Kansai Univ. 2001, and is in Master Course of Engineering of Osaka City Univ. 2001.

\section{Kazuhisa NIWA \\ Manager \\ Japan Information \\ Processing Service Co.,Ltd. Osaka, Japan}

Kazuhisa Niwa, born 1961, got his Doctor of Engineering from Kansai Univ. in 1999. He has an additional post, Part-time Assistant Professor of Kansai Univ.

\author{
Toshiyuki KITADA \\ Professor \\ Osaka City University \\ Osaka, Japan
}

Toshiyuki Kitada, born 1945, graduated from Osaka City Univ. 1968, and got his Doctor of Engineering form Osaka Univ. 1980.

\author{
Hiroshi MATSUDA \\ Associate Professor \\ Nagasaki University \\ Nagasaki, Japan
}

Hiroshi Matsuda, born 1957, graduated from Kyushu Institute of Technology 1980, and got his Doctor from Kyushu Univ. 1987.

\section{Summary}

In this paper, the system for visualizing of phenomenon up to the ultimate state of existing structures is developed in order to understand their mechanical properties and behaviors with ease. This system has three main sub-systems, the sub-system for obtaining and identifying 3D coordinates of a structure from 2D photo images, the sub-system for F.E.M. analysis to investigate its mechanical properties and behaviors, and the visualizing sub-system to easily understand the information obtained from the previous two sub-systems. This system uses the image-mapping technology for obtaining and identifying 3D coordinates from $2 \mathrm{D}$ photo images and $2 \mathrm{D}$ coordinates from 3D analytical results in reverse. And the applicability of the developed system by using a steel column specimen with box cross section is discussed.

Keywords: existing structure, failure mode, image information technology, image mapping technology, visualization

\section{Introduction}

For determining the retrofitting or strengthening method of existing structures designed by the past design code, it is very important to understand their current states, such as load carrying capacity under seismic motion. The most preferable and easiest method of investigating the current states is to carry out experiment, but it is impossible to destroy the existing structures.

Furthermore, engineering judgment by a skilled engineer might be needed to understand the current states of an existing structure and to determine the effective retrofitting or strengthening method, by using huge output data of numerical analysis. Accordingly, in order to determine quickly the effective retrofitting or strengthening method, development of a cheap and user-friendly simple system that can help engineers will be desired.

The objective of this study is to develop a useful system by using new information technologies which can visualize various phenomena of the existing structure and help the engineers to understand the current states of the structure. By using the system, various kinds of possible failure and damage of the structure can be predicted appropriately from the deformed images of the structure based on numerical results by finite element analysis. Moreover, it is very convenient if strain and stress contours can be also seen on the same deformed images. 


\section{System for visualizing various structural information}

This developed system consists of five sub-systems, as shown in Fig.1. The first sub-system is for recognizing $3 \mathrm{D}$ coordinates of the existing structure from the 2D photograph images. A key in this sub-system is mapping. The second sub-system is for generating image data to visualize many kinds of structural information. The third sub-system is for building an analytical model such as a finite element model by integrating material information and the 3D coordinates. The forth sub-system is for evaluating structural properties and behaviors such as the ultimate resistance force of the model analytically. In this sub-system, EPASS[1], USSP[2], and the other general-purpose F.E.M. programs[3] can be used. The last fifth sub-system is for visualizing the output results of the analysis by using the mapping technology.

\section{Obtaining of 3D coordinates of structures from 2D photograph images}

In this chapter, the most important first sub-system is explained focusing on how to obtain the 3D coordinates from 2D photograph images.

\subsection{Hardware composition}

To obtain the 3D coordinates from 2D photograph images, one digital camera and one calibration frame are utilized in the first sub-system.

\subsubsection{Digital camera}

In order to get $2 \mathrm{D}$ photograph image information, a digital camera is used in this system. Characteristics of the camera used are shown in Table 1. The reasons to use the digital camera are that it is easy to take photographs and to rectify them and the running cost is not expensive compared with an analog camera. But the analog camera can be used in this sub-system by digitizing with a scanner. However, distortion of the photograph by scanning must be removed.

\section{Table 1 Characteristics of camera used}

\begin{tabular}{|c|c|}
\hline Name & Canon Power Shot Pro70 \\
\hline Image Sensor Size \& Type & 0.5-inch CCD \\
\hline Resolution(Megapixels) & $1536 \times 1024$ \\
\hline Gradation Reproducibility & 24bit Color \\
\hline $\begin{array}{c}\text { Lens Focal Length } \\
\text { (35mm Equivalent Format) }\end{array}$ & $\begin{array}{c}6-15 \mathrm{~mm} \\
(28 \mathrm{~mm}-70 \mathrm{~mm}) \\
\end{array}$ \\
\hline
\end{tabular}

\subsubsection{Calibration frame}

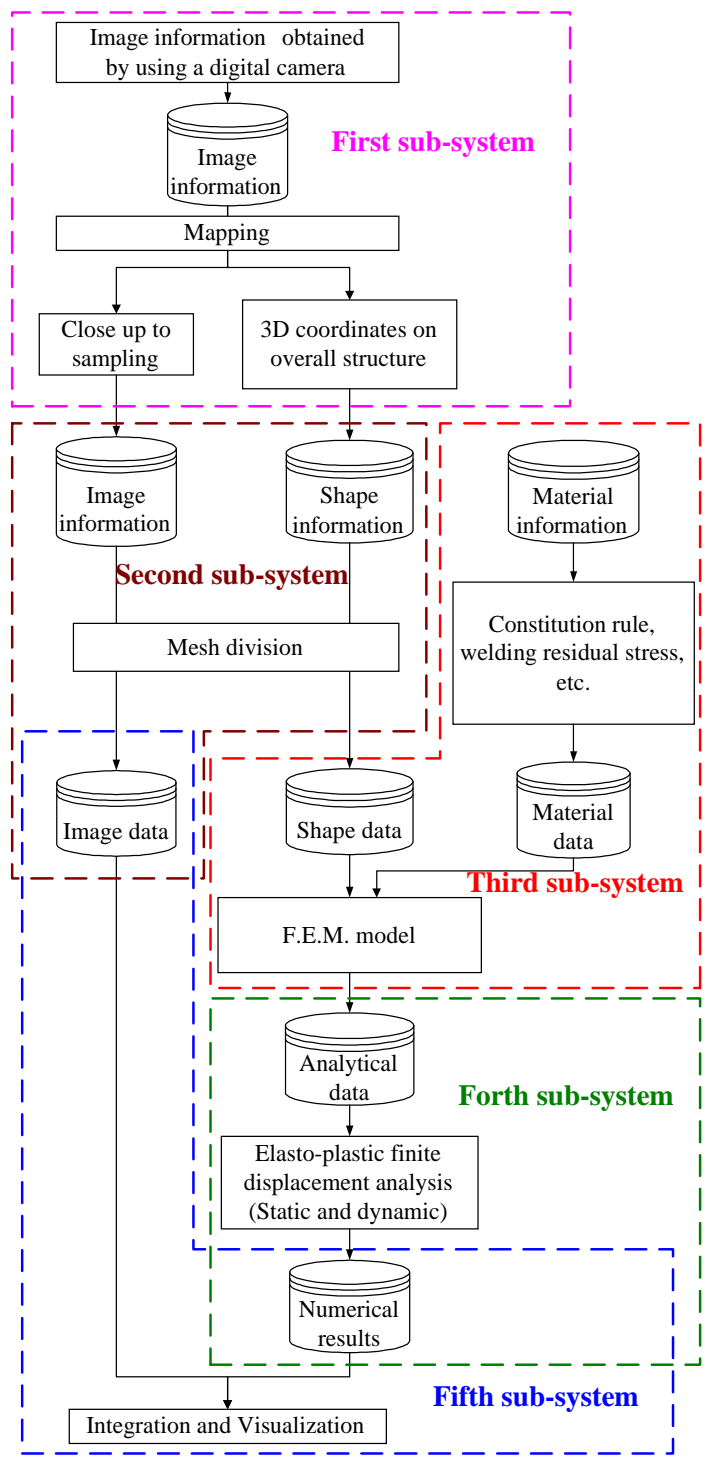

Fig.1 Outline of developed system

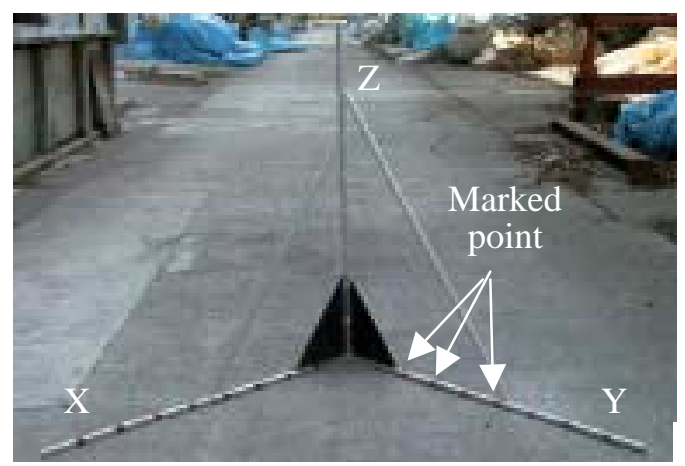

Fig.2 Calibration Frame

The calibration frame (henceforth C.F.) consists of three bars and is used to define the absolute coordinate system. 2D image photographs are taken with the C.F.. The C.F. used is shown in Fig.2. It is made of 3 aluminum bars of which length are $900 \mathrm{~mm}$. Three bars correspond to $\mathrm{x}$-axis, $\mathrm{y}$-axis, and $\mathrm{z}$-axis, respectively. Points are marked every $100 \mathrm{~mm}$. 


\subsection{Software composition}

The software for the first sub-system is developed in this study to obtain 3D coordinates from 2D photograph images.

The developed program has following two functions. The first function is for calculating a camera parameters using the acquired 2D coordinates of the specified points ( 9 points on C. F.) from the image. The second function is for calculating 3D coordinates using the acquired $2 \mathrm{D}$ coordinates of the specified points and calculated camera parameters. This program is coded by $\mathrm{C}++[4]$.

\subsubsection{Measurement theory}

A stereo pair images method based on the theory of triangulation[5] is used for the measurement.

If $3 \mathrm{D}$ coordinates $P_{i}\left(x_{i}, y_{i}, z_{i}\right)$ of a point on an object in the absolute coordinate system and 2D coordinates $P_{i}^{\prime}\left(u_{i}, v_{i}\right)$ corresponding to the point in the camera coordinate system are defined as shown in Fig.3, the perspective transformation can be done by the following Eq.(1).

$$
\lambda\left[\begin{array}{c}
u_{i} \\
v_{i} \\
1
\end{array}\right]=\left[\begin{array}{llll}
C_{11} & C_{12} & C_{13} & C_{14} \\
C_{21} & C_{22} & C_{23} & C_{24} \\
C_{31} & C_{32} & C_{33} & C_{34}
\end{array}\right]\left[\begin{array}{c}
x_{i} \\
y_{i} \\
z_{i} \\
1
\end{array}\right]
$$

The coefficient matrix, $\mathbf{C}$ of Eq.(1) is called the camera parameter matrix. They depend on the position of the camera in the absolute coordinate system, and the focal length of the lens of a camera. From Eq.(1), the constant, $\lambda$, can be written as

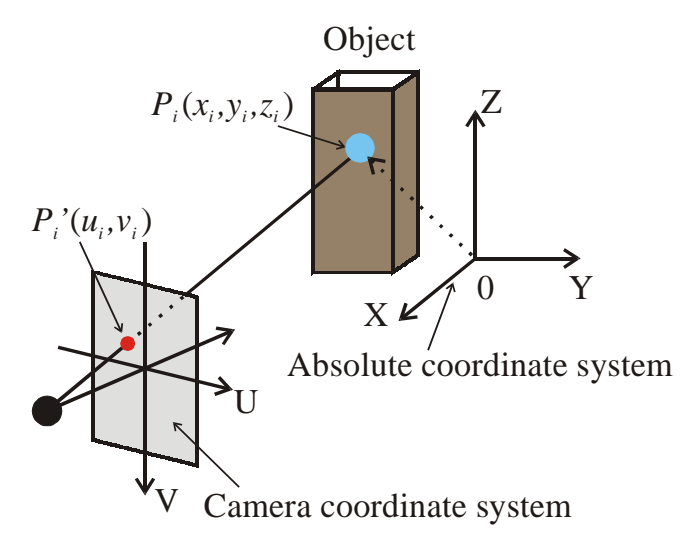

Fig.3 Perspective transformation

$$
\lambda=C_{31} x_{i}+C_{32} y_{i}+C_{33} z_{i}+C_{34}
$$

Substituting Eq.(2) to Eq.(1), the following Eq.(3) will be obtained.

$$
\left[\begin{array}{lll}
C_{11}-C_{31} u_{i} & C_{12}-C_{32} u_{i} & C_{13}-C_{33} u_{i} \\
C_{21}-C_{31} v_{i} & C_{22}-C_{32} v_{i} & C_{23}-C_{33} v_{i}
\end{array}\right]\left[\begin{array}{l}
x_{i} \\
y_{i} \\
z_{i}
\end{array}\right]=\left[\begin{array}{l}
C_{34} u_{i}-C_{14} \\
C_{34} v_{i}-C_{14}
\end{array}\right]
$$

If 3D coordinates of $P_{i}$ and 2D coordinates of $P_{i}$ ' are known, the following Eq.(4) is derived including the unknown $C_{i j}$ when $C_{34}$ assumes to be 1 .

$$
\left[\begin{array}{ccccccccccc}
x_{i} & y_{i} & z_{i} & 1 & 0 & 0 & 0 & 0 & -x_{i} u_{i} & -y_{i} u_{i} & -z_{i} u_{i} \\
0 & 0 & 0 & 0 & x_{i} & y_{i} & z_{i} & 1 & -x_{i} v_{i} & -y_{i} v_{i} & -z_{i} v_{i}
\end{array}\right]\left[\begin{array}{c}
C_{11} \\
C_{12} \\
\vdots \\
C_{33}
\end{array}\right]=\left[\begin{array}{c}
u_{i} \\
v_{i}
\end{array}\right]
$$

Therefore, since the number of the unknown camera parameters is 11 , more than 6 data points, which are not on the same one plane, are needed for calibration at least. If the calibration are carried out using $n$ data points, the following Eq.(5) will be obtained.

$$
\left[\begin{array}{ccccccccccc}
x_{1} & y_{1} & z_{1} & 1 & 0 & 0 & 0 & 0 & -x_{1} u_{1} & -y_{1} u_{1} & -z_{1} u_{1} \\
0 & 0 & 0 & 0 & x_{1} & y_{1} & z_{1} & 1 & -x_{1} v_{1} & -y_{1} v_{1} & -z_{1} v_{1} \\
\vdots & \vdots & \vdots & \vdots & \vdots & \vdots & \vdots & \vdots & \vdots & \vdots & \vdots \\
x_{n} & y_{n} & z_{n} & 1 & 0 & 0 & 0 & 0 & -x_{n} u_{n} & -y_{n} u_{n} & -z_{n} u_{n} \\
0 & 0 & 0 & 0 & x_{n} & y_{n} & z_{n} & 1 & -x_{n} v_{n} & -y_{n} v_{n} & -z_{n} v_{n}
\end{array}\right]\left[\begin{array}{c}
C_{11} \\
C_{12} \\
\vdots \\
C_{32} \\
C_{33}
\end{array}\right]=\left[\begin{array}{c}
u_{1} \\
v_{1} \\
\vdots \\
u_{n} \\
v_{n}
\end{array}\right]
$$


Eq.(5) is summarized as following,

$\mathrm{AC}=\mathbf{R}$

Camera parameters are derived from Eq.(7) using the least square method.

$\mathbf{C}=\left(\mathbf{A}^{\mathrm{t}} \mathbf{A}\right)^{-1} \mathbf{A}^{\mathrm{t}} \mathbf{R}$

where $\mathbf{C}$ is the camera parameter matrix.

Since two cameras are used as shown in Fig. 4 in a stereo pair image method, following simultaneous Eq.(8) will be written from Eq.(3).

$\left[\begin{array}{ccc}C_{11}^{L}-C_{31}^{L} u_{i}^{L} & C_{12}^{L}-C_{32}^{L} u_{i}^{L} & C_{13}^{L}-C_{33}^{L} u_{i}^{L} \\ C_{21}^{L}-C_{31}^{L} v_{i}^{L} & C_{22}^{L}-C_{32}^{L} v_{i}^{L} & C_{23}^{L}-C_{33}^{L} v_{i}^{L} \\ C_{11}^{R}-C_{31}^{R} u_{i}^{R} & C_{12}^{R}-C_{32}^{R} u_{i}^{R} & C_{13}^{R}-C_{33}^{R} u_{i}^{R} \\ C_{21}^{R}-C_{31}^{R} v_{i}^{R} & C_{22}^{R}-C_{32}^{R} v_{i}^{R} & C_{23}^{R}-C_{33}^{R} v_{i}^{R}\end{array}\right]\left[\begin{array}{c}x_{i} \\ y_{i} \\ z_{i}\end{array}\right]=\left[\begin{array}{c}C_{34}^{L} u_{i}^{L}-C_{14}^{L} \\ C_{34}^{L} v_{i}^{L}-C_{24}^{L} \\ C_{34}^{R} u_{i}^{R}-C_{14}^{R} \\ C_{34}^{R} v_{i}^{R}-C_{24}^{R}\end{array}\right]$

Where $C_{i j}^{L}$ and $C_{i j}^{R}$ denote the left and right camera parameters respectively. $P_{i}{ }^{L},\left(u_{i}{ }^{L}, v_{i}{ }^{L}\right)$ and $P_{i}{ }^{L},\left(u_{i}{ }^{R}, v_{i}^{R}\right)$ are 2D coordinates of the position of the left and right camera. Above Eq.(8) is simplified using matrix expressions,

$\mathbf{B P}=\mathbf{S}$

Therefore, finally, 3D coordinates of $P_{i}$ can be obtained from the following Eq.(10).

$\mathbf{P}=\left(\mathbf{B}^{\mathrm{t}} \mathbf{B}\right)^{-\mathbf{1}} \mathbf{B}^{\mathrm{t}} \mathbf{S}$

3.2.2 Compensation of distortion of $2 \mathrm{D}$ coordinates from digital camera

The lens of the camera has certain distortion in general. Such distortion is called as the lens distortion here. It affects greatly the evaluation of 3D coordinates [6]. Fig.5 shows the influence of the lens distortion. Both the actual 2D coordinates of a graph paper and the calculated 2D coordinates after measuring are shown in Fig.5 for comparison. The former is drawn by the red lines, and the latter is drawn by the blue lines and the frame of the camera is drawn by the green lines.

It can be found that the further the point is from the center point, the larger the gap becomes. Therefore, in order to obtain accurate 3D coordinates from digital camera images, distortion of the lens should be removed by compensation of adjusting camera parameters.

In this study, 2D coordinates obtained to calculate both camera parameters from points on C.F. and 3D coordinates from an object are compensated.

\section{Application example of steel column specimen}

\subsection{Column specimen under consideration}

In order to investigate the applicability of the developed system, a steel column specimen with box cross section is used. The specimen used is shown in Fig.6. Geometrical configurations of the specimen are depicted in Fig.7. In this figure, the dimensions are the design values.

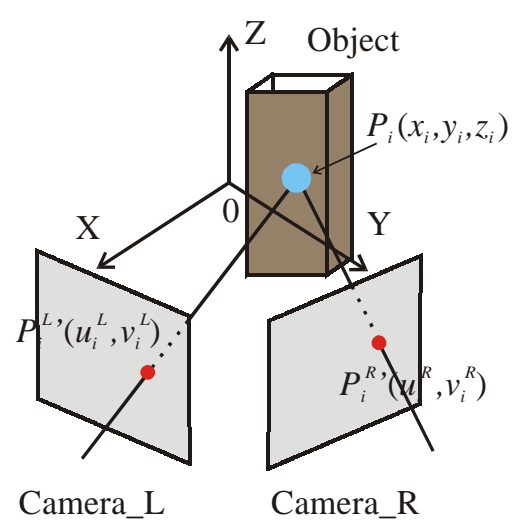

Fig.4 Stereo pair images method
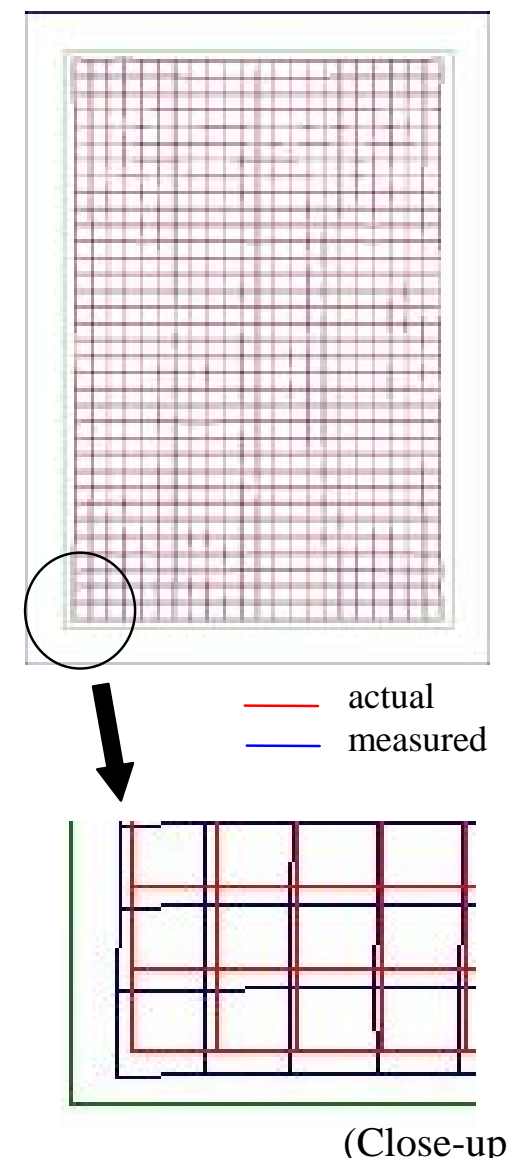

Fig.5 Comparison of actual dimension with measured dimension 
Many lines every $50 \mathrm{~mm}$ along both the horizontal and vertical directions are drawn on the specimen as shown in Fig.6 to mark the points which are used in mapping.

\subsection{Identification of $3 \mathrm{D}$ coordinates}

At first, photographs are taken from different 2 directions focusing on each corner of the specimen as shown in Fig.8. And then, 3D coordinates of each corner section are identified from these taken photo images respectively using a stereo pair images method mentioned above.

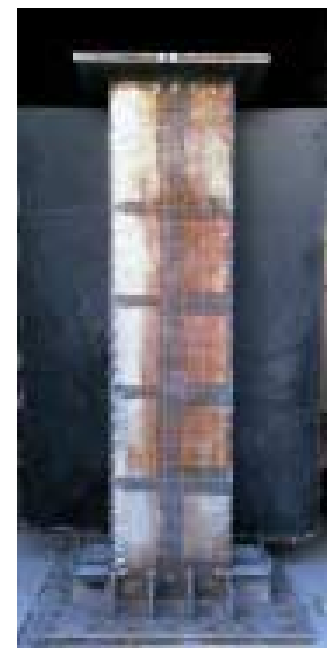

Fig.6 Steel column specimen

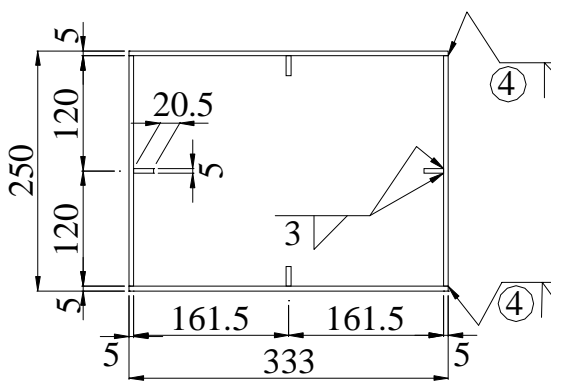

(a) Cross section

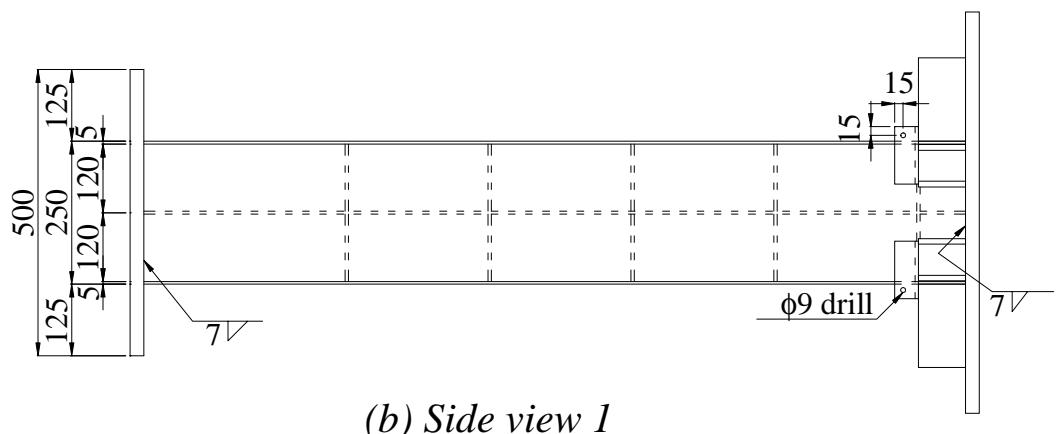

(b) Side view 1

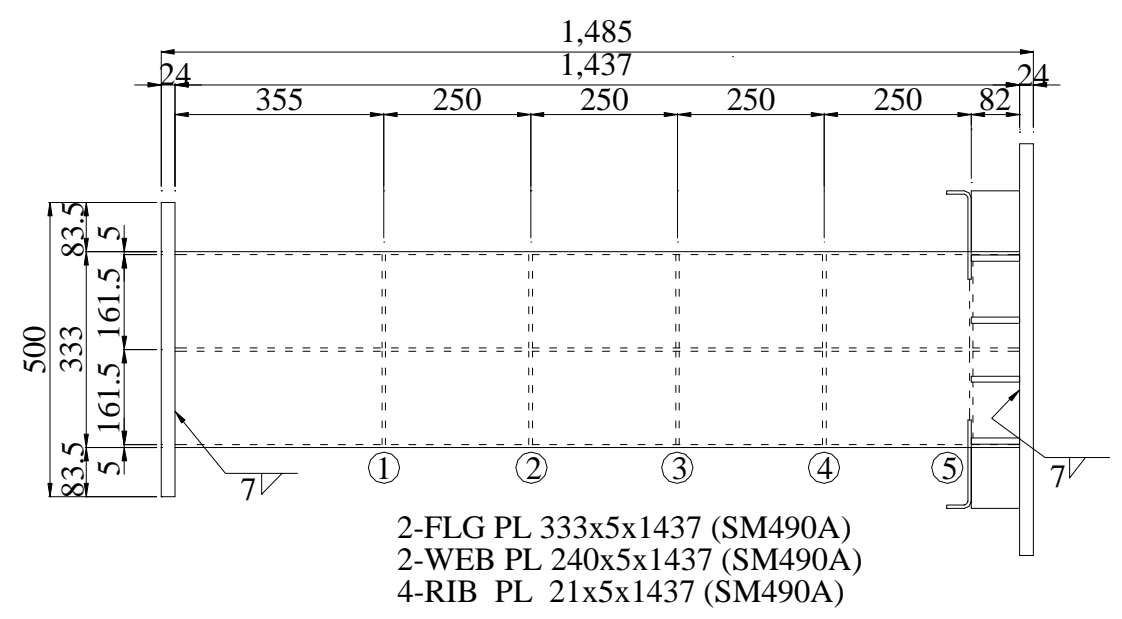

(c) Side view 2

Fig.7 Geometrical configurations of the steel column specimen

(Unit:mm)

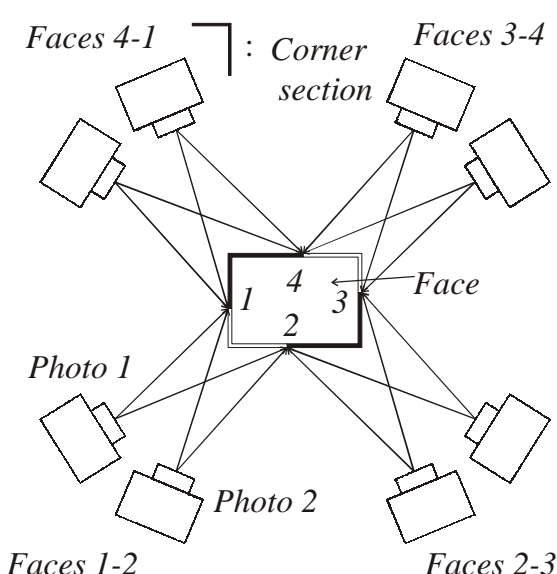

(a) Locations at a digital camera

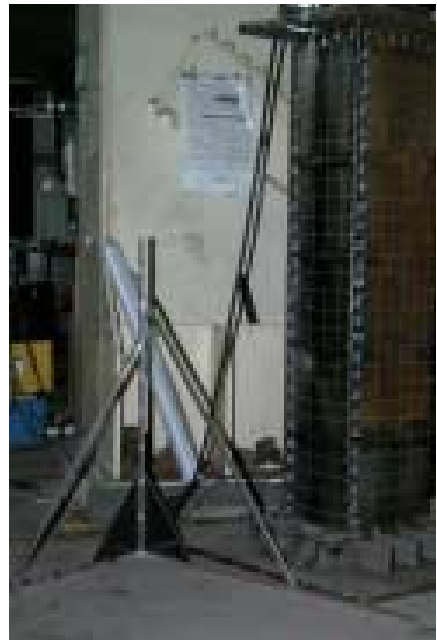

(b) Photo 1

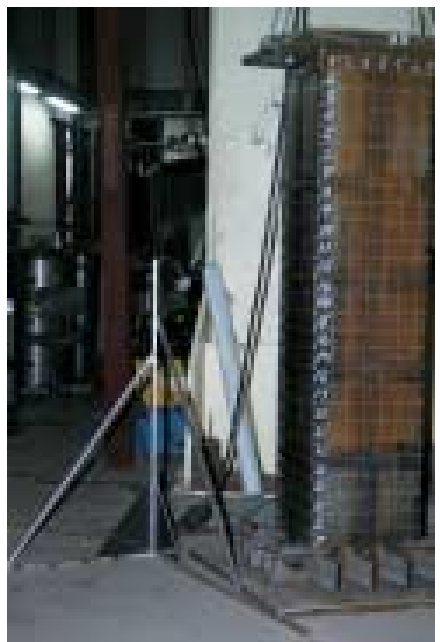

(c) Photo 2

Fig.8 Photography method for obtaining 3D coordinates of each corner 


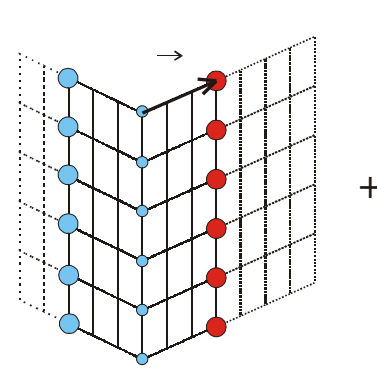

Faces 1-2

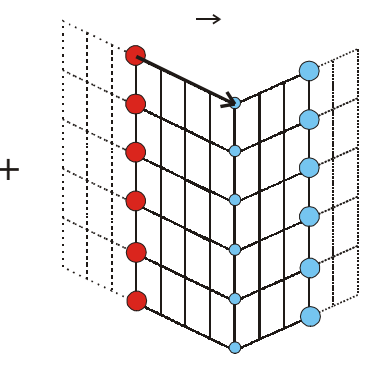

Faces 2-3

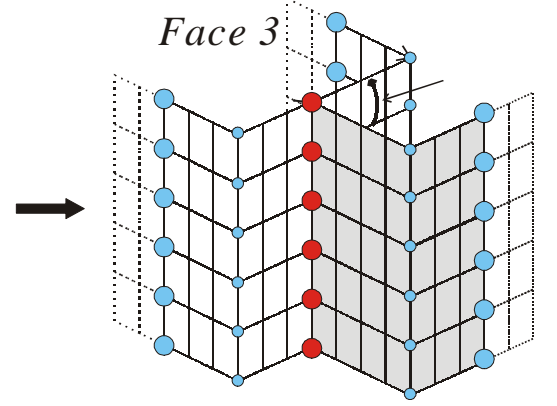

Face 1 Face 2 Face 3

Fig.9 Method of combining 4 faces

Secondary, 3D coordinates of the whole target points on the specimen are determined by using the 3D coordinates of each corner sections as shown in Fig.9. The four box surfaces are defined as Facel to Face 4 in the figure. The corner section of Facel and Face2 is called as Face1-2. Since obtained 3D coordinates of each corner section has individual coordinates system, it is necessary to transfer them to the same one coordinates system. In this study, the four faces are combined by rotating the calculated angle $\theta$ formed by two vectors, $\vec{\alpha}$ and $\vec{\beta}$ which show the direction of the faces as shown in Fig.9.

However, 3D coordinates of the points on a corner line of a face are not coincided with their corresponding target points on the corner line of the neighboring face nevertheless they should have same 3D coordinates, because there are some errors in measuring and obtaining 3D coordinates. Therefore, the coordinates of the connect points are modified by using an error correct method to solve the problem for closed traverse.

\subsection{Measurement and Identification results}

Identified measurement results before combining the four faces are summarized in Tables 2 and 3. And, those after combining them are also listed in Tables 4 and 5. The specimen after the identification of 3D coordinates is shown in Fig.10. In these tables, the errors of the identified length to the actual length for Face 3 are listed. The average measurement value means the average of the whole calculated lengths of lines on the Face 3 obtained from 2D photo images.

The error of length, $e$, is defined by the following Eq.(11).

$e=\left|\frac{A-B}{A}\right| \times 100$

where $A$ is the actual length measured directly, $B$ is the identified length from $2 \mathrm{D}$ photo images.

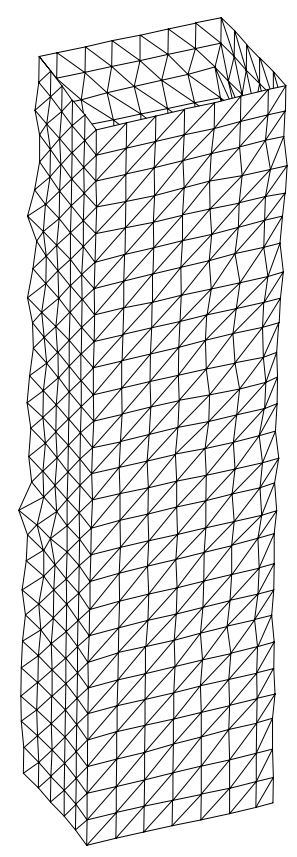

Fig.10 Specimen combined
Table 2 Accuracy in the width direction before combining

\begin{tabular}{|c|c|c|c|}
\hline & actual & average & eroors \\
\hline normal & \multirow{2}{*}{250.0} & 262.1 & $4.84 \%$ \\
\cline { 1 - 3 } \cline { 3 - 4 } compensated & & 251.0 & $0.39 \%$ \\
\hline
\end{tabular}

Table 4 Accuracy in the width direction after combining

\begin{tabular}{|c|c|c|c|}
\hline & actual & average & eroors \\
\hline normal & \multirow{2}{*}{250.0} & 260.6 & $4.25 \%$ \\
\cline { 3 - 4 } compensated & & 246.7 & $-1.31 \%$ \\
\hline
\end{tabular}

Table 3 Accuracy in the height direction before combining

\begin{tabular}{|c|c|c|c|}
\hline & actual & average & eroors \\
\hline normal & \multirow{2}{*}{1355.0} & 1372.1 & $1.26 \%$ \\
\cline { 1 - 3 } compensated & & 1357.2 & $0.17 \%$ \\
\hline
\end{tabular}

Table 5 Accuracy in the height direction after combining

\begin{tabular}{|c|c|c|c|}
\hline & actual & average & eroors \\
\hline normal & \multirow{2}{*}{1355.0} & 1356.5 & $0.11 \%$ \\
\cline { 3 - 4 } compensated & & 1344.5 & $-0.78 \%$ \\
\hline
\end{tabular}

(Unit : $\mathrm{mm}$ ) 
It is found that the accuracy of both width and height directions is improved by compensating the lens distortion before the combining. Length after the combining tends to shorter than the length before the combining. It is caused by the assumption that $z$ coordinates are common among all the measuring points where located on a certain horizontal line. For more improvement of the accuracy, it should be considered that $z$ coordinates are different in original.

\subsection{Visualized output results}

In this section, the fifth sub-system, namely the system for visualizing the analytical results is discussed.

In order to obtain the deformed states of the specimen, F.E.M. elastic analysis subjected to monotonic loading are carried out. The analysis is the elastic analysis because the main object is only to obtain the deformed state for checking the applicability of the developed system. Boundary and loading conditions are shown in Fig.11. It is necessary to input the material information, because the information cannot be only obtained from the photo image. The material properties used for the analysis are tabulated in Table 6.

Table 6 Material properties

\begin{tabular}{|c|c|}
\hline yield strength & $44.87(\mathrm{kN})$ \\
\hline yield stress & $371.67\left(\mathrm{~N} / \mathrm{mm}^{2}\right)$ \\
\hline Young's modulus & $2.12 \times 10^{5}\left(\mathrm{~N} / \mathrm{mm}^{2}\right)$ \\
\hline Poisson's ratio & 0.28 \\
\hline
\end{tabular}

Finite elements used in the analysis are triangle elements and the nodes of the elements coincide with $3 \mathrm{D}$ coordinates of the target points. The deformation of the specimen based on the analytical results is shown in Fig. 11 .

By using the mapping technology, it is attempted to show the deformation of the specimen on the same photograph image which is taken for obtaining 3D coordinates. The results are shown in Fig.12 and 13. Fig.12 shows the initial state before the deformation, and Fig.13 shows the deformed state.

It will be found that the deformation of the specimen can be understand easily on the $2 \mathrm{D}$ photo image by using the developed system.

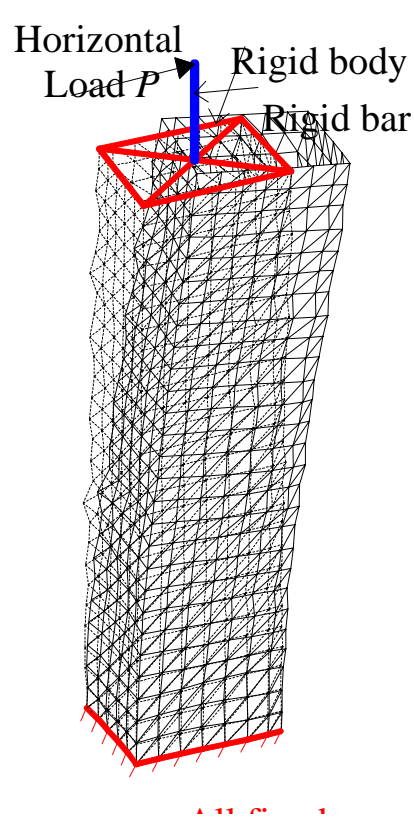

Fig.11 Deformed state by F.E.M. analysis

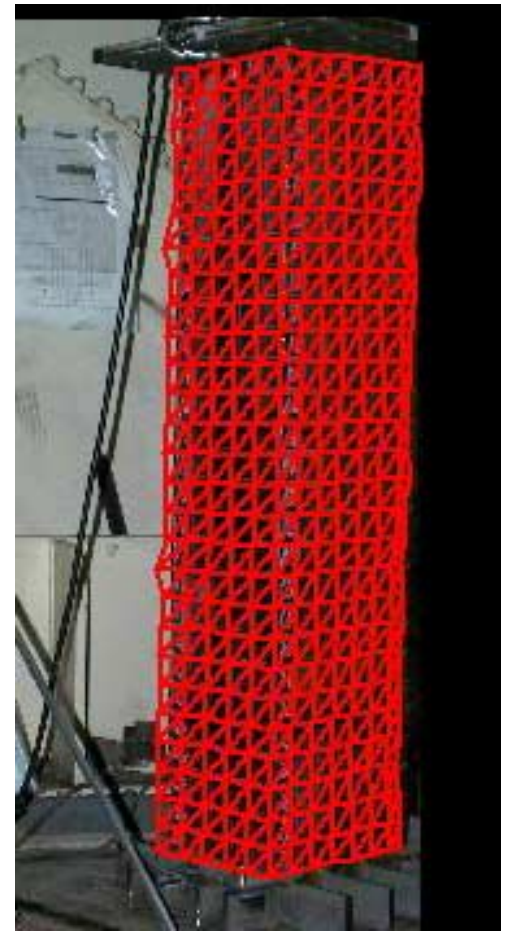

Fig.12 Identified 3D coordinates on the $2 D$ photograph (Initial state before loading)

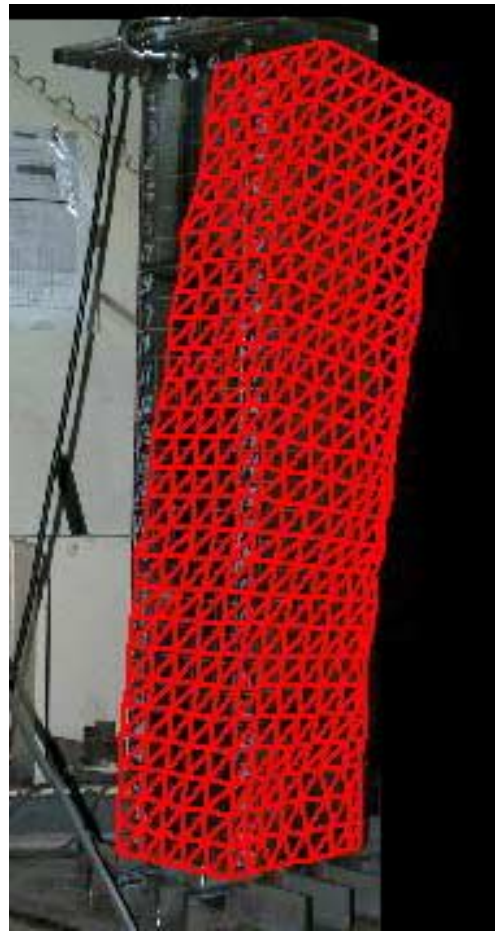

Fig.13 Identified 3D coordinates on the $2 D$ photograph

(Deformation: 10 times the real dimension) 


\section{Concluding remarks and future research needs}

In this paper, the $3 \mathrm{D}$ coordinates were identified based on the $2 \mathrm{D}$ image photos obtained with a digital camera. Then, elastic analysis for an analytical model made using the identified 3D coordinates of the specimen are performed. Moreover, the analytical results were reflected in the 2D original photo image to make the realistic deformed states of an existing structure under consideration visually for easy understanding of the structural properties and behaviors. Main conclusions obtained in this study are as follows:

1) The visualizing system for easy understanding of the mechanical properties and behaviors of existing structures are developed using the information technology, such as the identification of $3 \mathrm{D}$ coordinates and visualizing the deformed states of a specimen on the original 2D photo images. And it is found that the visualizing is possible, effective, easy and not expensive by using this developed system. Moreover, taking into account for portability of the developed system, the measurement on the fields is possible as compared with the current measurement systems.

2) Distortion due to lens is not neglected in photographs taken by a camera. However, proposed compensation of the distortion can be mitigated to the extent that the problem is negligible.

The following issues should be improved in the future.

1) In order to identify 3D coordinates with more accuracy, using a digital camera with higher resolution, developing the combined methods for building up the structure from some portions and methods of taking photos should be considered

2) A convenient method for obtaining invisible material information needed in the analytical part, such as material properties, thickness of plates and position of stiffeners, which cannot be obtained from the 2D photo images directly, should be devised in the developed system.

3) Although the deformation of a structure can be expressed visually with CG using analytical results in the developed system, not only deformation but also other much information, such as colors considering the shadow of the surface must be visualized realistically. Furthermore, to show the properties and the behaviors of the existing structures effectively, 3D virtual image producing system will be put in the developed system.

\section{References}

[1] KITADA T., OHMINAMI R., NIBU M., and TANAKA K., "Exploitation of Computer Program to Analyze Ultimate Strength of Steel Bridge Using Cable Members", Proceedings of Symposium on Computational Methods in Structural Engineering and Related Fields, JSSC, Vol.13, pp.89-94, July 1989 (in Japanese).

[2] KOMATSU S., and KITADA T., "Elasto-Plastic Finite Displacement Analysis of Longitudinally Stiffened Plates in Compression", Proceedings of JSCE, No.296, pp.1-12, April 1980 (in Japanese).

[3] HIBBIT, KARLSSON, and SORENSEN, Inc.: ABAQUS/Standard User's Manual, Vol.I-III Version 5.4, 1995

[4] MATSUDA H., WADA M., KOJIMA S., SAKIYAMA T., MORITA C., MORIYAMA M., KOGA A., NAKAMURA M., YAMAMOTO A., and TSURUTA K., "Development of 3D measuring system and applications to construction joints of concrete and to free vibration analysis of twisted cylindrical panel" Journal of applied mechanics, JSCE, Vol.3, pp.115-124, September 2000 (in Japanese).

[5] INOGUCHI S., and YAMASHITA T.: 3D Imaging Techniques for Measurement, Shokodo, November 1990 (in Japanese).

[6] MATSUDA H., KOJIMA S., WADA M., KOGA A., and NAKAMURA M., "Development 3D measurement and FEM analysis system for membrane and shell structures" Journal of Constructional Steel, JSSC, Vol.8, pp.595-602, November 2000 (in Japanese). 